Bio - grafía. Escritos sobre la Biología y su Enseñanza. ISSN 2027-1034

Edición Extraordinaria. p.p. 1403 - 1414

Memorias del IX Encuentro Nacional de Experiencias en Enseñanza de la Biología y la Educación Ambiental. IV Congreso Nacional de Investigación en Enseñanza de la Biología.

\title{
EL CONOCIMIENTO PROFESIONAL DE DOCENTES PRINCIPIANTES DE CIENCIAS NATURALES: UN ESTUDIO CON PROFESORES DE DEL DEPART AMENTO DEL HUILA
}

\section{THE PROFESSIONAL KNOWLEDGE OF TEACHERS BEGINNERS OF NATURAL SCIENCES, APROPOSAL AT THE SURCOLOMBIAN UNIVERSITY}

\author{
Mosquera, Jonathan Andrés ${ }^{1}$ \\ Amórtegui Cedeño, Elías Francisco ${ }^{2}$
}

\section{RESUMEN}

Presentamos el diseño de instrumentos y aplicación preliminar para el primer proyecto de investigación en el departamento del Huila, en torno a la configuración del Conocimiento Profesional del Profesor de ciencias naturales, en el contexto de docentes principiantes graduados de la Universidad Surcolombiana. Este estudio se enmarca en un diseño de investigación mixto, de tipo descriptivo e interpretativo, en donde hacemos uso de análisis cualitativos para caracterizar el proceso de inserción profesional de los docentes, sus concepciones y producciones de aula, que contribuyen a consolidar un conocimiento de referencia, y de análisis cuantitativos para correlacionar variables del cuestionario principal, en donde hemos incorporado preguntas de contexto abierto, de selección múltiple y de valoración, acorde a un inventario de preocupaciones y problemas para docentes principiantes. La población de estudio, se divide en dos partes, 110 graduados de la Licenciatura en Ciencias Naturales de la Universidad Surcolombiana, entre los años 2012 a 2017 para la fase descriptiva, y 2 casos voluntarios para la fase interpretativa, casos que llevan poco tiempo de inserción laboral y máximo se han graduado hasta 3 años atrás. Aunque se ha profundizado en la temática a nivel regional, el campo de estudio sigue poco explorado, lo que nos motiva a continuar, dado que a nivel institucional y en atención a políticas de acreditación de alta calidad, el programa y la universidad, requiere valorar el impacto de sus graduados, los proceso de inserción laboral y los cambios que los profesores de ciencias naturales asumen en torno a su saber profesional.

PALABRAS CLAVE: Docentes principiantes, inserción docente, Conocimiento Profesional del Profesor, preocupaciones y problemas.

\footnotetext{
${ }^{1}$ Docente de Cátedra, Universidad Surcolombiana, jonathan.mosquera@usco.edu.co Grupo de Investigación Conocimiento Profesional del Profesor de Ciencias - CPPC

${ }^{2}$ Docente de Planta Tiempo Completo, Universidad Surcolombiana, elias.amortegui@usco.edu.co Grupo de Investigación Conocimiento Profesional del Profesor de Ciencias - CPPC
} 
Bio - grafía. Escritos sobre la Biología y su Enseñanza. ISSN 2027-1034

Edición Extraordinaria. p.p. 1403 - 1414

Memorias del IX Encuentro Nacional de Experiencias en Enseñanza de la Biología y la

Educación Ambiental. IV Congreso Nacional de Investigación en Enseñanza de la Biología.

\begin{abstract}
We present the instruments design and preliminary application for the first research project in the department of Huila, around the configuration of the Professional Knowledge of the Professor of Natural Sciences, in the context of beginner teachers graduated from the Surcolombiana University. This study is part of a mixed research design, descriptive and interpretive, where we make use of qualitative analysis to characterize the process of professional insertion of teachers, their conceptions and classroom productions, which contribute to consolidate a reference knowledge, and quantitative analysis to correlate variables of the main questionnaire, where we have incorporated open context, multiple selection and assessment questions, according to an inventory of concerns and problems for beginner teachers. The study population is divided into two parts, 110 graduates of the Degree in Natural Sciences of the Surcolombiana University, between the years 2012 to 2017 for the descriptive phase, and 2 volunteer cases for the interpretative phase, cases that take little time to Labor insertion and maximum have graduated up to 3 years ago. Although the subject has been deepened at the regional level, the field of study remains little explored, which motivates us to continue, given that at the institutional level and in light of high quality accreditation policies, the program and the university, the impact of their graduates, the processes of labor insertion and the changes that professors of natural sciences assume around their professional knowledge.
\end{abstract}

KEY WORDS: Beginning teachers, teacher insertion, Professional Knowledge of the teacher, concerns and problems.

\title{
INTRODUCCIÓN
}

El estudio en torno al Conocimiento Profesional del Profesor, ha venido profundizando en diferentes líneas académicas, son varios los autores que al respecto han hecho grandes aportes a la comprensión del tema (Shulman, 1986, 1987; Bromme, 1988; Tardif, 2004; Grossman, 1990; Marcelo, 1999; Valbuena, 2007; Amórtegui, Correa \& Valbuena, 2009; Amórtegui, 2011; Amórtegui et al., 2015, 2016 y Castrillón et al., 2016), sin embargo estos estudios sobre el Conocimiento Profesional del Profesor (CPP) se han centrado en dos grandes tendencias, que son: la caracterización y ubicación sobre el CPP y el reconocimiento del Conocimiento Didáctico del Contenido (CDC). De esta manera y a pesar de este panorama, el Conocimiento Profesional del Profesor ha sido poco cuestionado desde la perspectiva del docente principiante. Esta población objeto de estudio cobra importancia, cuando reconocemos que los docentes que se gradúan de los programas de pregrado, se denominan docentes en ejercicio, ingresando a nuevos espacios de construcción de su conocimiento, reconociendo la realidad del sector laboral y enfrentando las características del contexto de aula y escuela (Jiménez, 2013). En algunas ocasiones la inserción puede ser amable, aunque en otras, el proceso provoca en cadena un efecto dominó de emociones encontradas, que siempre acaban en la 


\title{
Bio - grafía. Escritos sobre la Biología y su Enseñanza. ISSN 2027-1034
}

\section{Edición Extraordinaria. p.p. 1403 - 1414}

\author{
Memorias del IX Encuentro Nacional de Experiencias en Enseñanza de la Biología y la \\ Educación Ambiental. IV Congreso Nacional de Investigación en Enseñanza de la \\ Biología.
}

configuración de la construcción de un conocimiento específico, para el docente en ejercicio.

Algunos investigadores considerar que los primeros años de la carrera de un profesor de ciencias están marcados por las creencias que cambian rápidamente e indicen de manera espontánea pero decisiva, sobre los conocimientos y las prácticas educativas del mismo (Davis, Petish \& Smithey, 2006; Luft et al., 2011). Para Freire y Fernandez (2015), a pesar de contar con una creciente producción de conocimiento en los últimos años, esta rama de la investigación educativa está muy distante de la meta, cuando se tiene en cuenta todos los cambios que sufre un maestro en su incorporación al mundo laboral, su ubicación en un centro educativo (escuela, colegio, universidad) y su adaptación al contexto. En el contexto nacional, destacamos el trabajo de Ramírez (2016), en donde se identificaron los principales problemas que afrontaron los docentes novatos graduados de diferentes Instituciones de Educación superior en Colombia. Para el contexto en particular del Departamento del Huila, registran estudios como el Amórtegui et al. (2016) y Bernal et al. (2016) con futuros docentes que cursaban su práctica pedagógica, y el trabajo de Castrillón et al. (2016), con docentes en formación inicial que cursaban sus primeros espacios sobre didáctica de las ciencias naturales.

\section{METODOLOGÍA}

En esta investigación el problema de estudio se abordará desde un enfoque mixto, en el cual se combinan aspectos de la metodología cuantitativa y cualitativa (Creswell, 2005), teniendo en cuenta un diseño descriptivo e interpretativo, que permite explorar el problema de estudio en este caso particular en relación a la configuración de un conocimiento profesional específico por parte de docentes principiantes. El diseño investigativo es de dos etapas, la primera de tipo descriptivo (cuantitativa) y la segunda interpretativa (cualitativa). Según Hernández, Fernandez y Baptista (2006), el diseño descriptivo es no experimental, de corte transeccional, y en el interpretativo se tendrá en cuenta el diseño fenomenológico, enfocando las experiencias de los docentes principiantes.

Para el desarrollo del proyecto se ha seleccionado como población de interés a los graduados de la Licenciatura en Ciencias Naturales de la Universidad Surcolombiana, que se hayan graduado entre febrero de 2012 y febrero de 2017, para un total de 158 docentes. Al validar la muestra estadística, se seleccionaron aleatoriamente 110, haciendo distinción entre sexo (61 mujeres y 49 hombres), para el análisis correlacional de la parte descriptiva.

En la recolección de información, adaptamos con autorización el cuestionario aplicado por Ramírez (2016), teniendo en cuenta el Inventario de Preocupaciones de Profesores formulado por Marcelo (1991), que consta de 42 items que responden a siete dimensiones: No Preocupación, Información, Personal, Gestión, Alumnos, Colaboración y Renovación. De igual forma, se relacionan algunas afirmaciones del listado de problemas propuesto por Veenman (1984). Aclaramos que los instrumentos fueron adaptados al 


\title{
Bio - grafía. Escritos sobre la Biología y su Enseñanza. ISSN 2027-1034
}

\section{Edición Extraordinaria. p.p. 1403 - 1414}

\author{
Memorias del IX Encuentro Nacional de Experiencias en Enseñanza de la Biología y la \\ Educación Ambiental. IV Congreso Nacional de Investigación en Enseñanza de la \\ Biología.
}

contexto de la investigación y se incorporaron algunos elementos que enfatizan en la experiencia de Práctica Pedagógica de cada docente, pues esto servirá a la triangulación posterior entre los resultados de la inserción profesional y la formación inicial en la construcción de un conocimiento de referencia.

Para la parte interpretativa, entre los docentes principiantes se seleccionaron 2 casos de manera voluntaria y bajo consentimiento informado para realizar un seguimiento durante 2 meses a la actividad docente en las Instituciones Educativas en donde se han insertado profesionalmente. Para esto se hace uso de diferentes técnicas para recolección de información, tales como: observación participante, grabación de clases, entrevistas semiestructuradas antes y después del seguimiento, formatos de reflexión, análisis documental de los documentos de las Instituciones Educativas, sobre el proceso de acompañamiento a los docentes en su inducción y acoplamiento laboral, y transcripciones de secuencias de clase, para hacer revisión del discurso de los docentes. La sistematización de la información recolectada, la realizaremos mediante la técnica del análisis de contenido (Pinto y Gálvez, 1996) en el software Atlas. Ti 7.0; teniendo en cuenta las categorías propuestas por Valbuena (2007) y Park y Chen (2012). De igual forma para complementar el análisis descriptivo, se hará el análisis correlacional a partir de los resultados de la escala sobre las preocupaciones y la escala de problemas que presentan los docentes principiantes durante la inserción profesional, haciendo uso de las dimensiones que componen cada una y siguiendo los postulados para esta revisión, propuestos por Reyes (2011).

\section{RESULTADOS}

En este apartado, mostramos la estructura de los principales instrumentos y los resultados preliminares de su aplicación, hacemos énfasis en el primer acercamiento a uno de los casos de la fase interpretativa, la docente principiante que para esta investigación se denominará Ana.

\section{Aplicación de Instrumentos}

El cuestionario empleado consta de seis (6) apartados, que se presentan a continuación: información personal, en donde se indagan aspectos como nombre (voluntario) o pseudónimo, sexo y edad, estos últimos para hacer análisis posteriormente entre estas variables y los problemas planteados por los docentes principiantes. Información Académica, se cuestiona por el programa del cual fue titulado, dado que la Licenciatura en Ciencias Naturales de la Universidad Surcolombiana ha tenido dos denominaciones, además se indaga por estudios post-graduales y la valoración que hace el docente de la formación recibida en la licenciatura. Información sobre la Práctica Pedagógica, institución donde adelantó la práctica, la asignatura abordada, el tipo de actividades que realizó, los productos académicos logrados o generados de esa experiencia, y la valoración del aporte de cooperador, asesor y demás elementos del curso para su formación como docente y su vida profesional, este apartado lo hemos incluido en el estudio, teniendo en cuenta los resultados mostrados en los trabajos de Amórtegui et al. (2015, 2016). 
Bio - grafía. Escritos sobre la Biología y su Enseñanza. ISSN 2027-1034

Edición Extraordinaria. p.p. 1403 - 1414

Memorias del IX Encuentro Nacional de Experiencias en Enseñanza de la Biología y la

Educación Ambiental. IV Congreso Nacional de Investigación en Enseñanza de la Biología.

Información Laboral, en donde se plantean interrogantes sobre la situación laboral actual del graduado, el tipo de institución en donde trabaja, el cargo, las asignaturas que orienta, el nivel en el cual labora y algunos aspectos del mecanismo experimentado para incorporarse al ámbito laboral y específicamente al educativo.

Por otro lado y como eje del estudio, se incorporan elementos en relación a la Información del proceso de inserción, a manera de relato, se cuestiona al docente sobre algunos recuerdos de sus primeros años de docencia, su primera experiencia de aula y los factores que influyeron en sus decisiones sobre la planificación y secuencias, y los procesos de acompañamiento que pudo haber hecho la institución educativa en el proceso de inserción. Tipos de problemas que afectaron su inicio en la docencia, corresponde a una escala tipo Likert en donde se presentan situaciones de aula y posibles problemas de inserción docente, para que cada profesor seleccione una postura y valore de 1 a 5 cada uno de los enunciados, teniendo en cuenta que 1 no representa ni ha representado ningún problema y 5 representa o ha representado un gran problema en su ejercicio docente.

Este cuestionario fue en un inicio piloteado con 6 docentes egresados de la licenciatura, externos a la población y muestra. Los docentes, leyeron cada pregunta y de presentar confusión en su escritura o no ser relevante desde su experiencia (entre 5 y 10 años de ejercicio en el aula), informaron al final del instrumento en el apartado de observaciones. Dentro de las principales apreciaciones encontramos:

D3.C1. [Haciendo relación a la forma del cuestionario] "el cuestionario es muy completo e interesante, considero que aportará significativamente a la autoevaluación del programa y de la universidad, revisando el potencial de nosotros los egresados".

D5.C1. [Haciendo relación al contenido del cuestionario] "considero que aunque es largo, es muy pertinente, incorpora elementos desde la práctica que en algunas ocasiones, no las recordamos, pero que aportan al ejercicio como docentes, espero que los resultados nos los puedan dar a conocer".

D6.C1. [Haciendo relación a la forma del cuestionario] "incorporar en un futuro o en otras investigaciones preguntas que no sean exclusivas a egresados que ejerzan la docencia, estamos otros que no únicamente estamos en el aula de clases".

Seguidamente, fue validado por 2 expertos y conocedores del tema de la formación de profesorado, el Jefe de Programa de la Licenciatura de la cual se graduaron nuestros profesores objeto de estudio, en la Universidad Surcolombiana, y una Docente Investigadora de la Universidad Pedagógica y Tecnológica de Colombia, con amplios 


\title{
Bio - grafía. Escritos sobre la Biología y su Enseñanza. ISSN 2027-1034
}

\section{Edición Extraordinaria. p.p. 1403 - 1414}

\author{
Memorias del IX Encuentro Nacional de Experiencias en Enseñanza de la Biología y la \\ Educación Ambiental. IV Congreso Nacional de Investigación en Enseñanza de la \\ Biología.
}

conocimientos en torno a la profesionalización del docente y su conocimiento propio y diferente a los demás profesionales.

En la actualidad estamos terminando de recopilar los últimos datos con este instrumento, conservando las proporciones estadísticas para distinción de sexo de los docentes principiantes. Encontrando que sólo el 60\% (30 de 50 a la fecha) está vinculado con el sector oficial de la educación, $36 \%$ en calidad de planta y el restante, 12 profesores en plaza provisional, en secretarías de educación como Huila (6), Neiva (2), Bogotá D.C. (2), Santa Marta (1) y Caquetá (1). El otro $40 \%$ se distribuye entre, sector privado (32\%) en municipios como Neiva, Pitalito y Garzón, y $8 \%$ que se distribuye entre 2 graduados que están adelantando actividades formativas posgraduales, y otros 2 que ejercen la docencia universitaria.

Para el factor de año de graduación, la toma de datos aleatoria ha sido significativa, pues se ha logrado abarcar, 10 docentes del año 2012, 6 del 2013, 4 para 2014, 14 del 2015, 12 de 2016 y finalmente 4 casos más recientes de la promoción enero de 2017, que ya están inmersos en un campo laboral.

A nivel de educación posgradual, sólo el 32\% (16 docentes) están o han cursado un programa después de su pregrado, informando el nivel de maestría. El $75 \%$ de los docentes, lo hacen o lo han hecho en programas presenciales, el $12.5 \%$ semipresencial y el $12.5 \%$ a distancia. Los programas más comunes, son la maestría en educación que oferta la Universidad Surcolombiana y la de Ingeniería y Gestión Ambiental, de la misma casa de estudios. Sin embargo es significativo que registran estudios en el exterior como el Mestre em Genética Evolutiva e Biologia Molecular, de la Universidad Federal de Sao Carlos en Brasil, que además la docente reportó ser producto de una beca. En otros estudios se reportan cursos de inglés, en atención a necesidades personales y políticas actuales en la educación, que tienden a planes de acción como el programa de bilingüismo.

Al indagar en torno a los procesos pedagógicos y las bases recibidas en el programa del cual se graduaron, los docentes exponen casos como:

D1.C1.P2. [Haciendo relación a la formación que recibió en su Universidad] "Una formación excelentes, con bastante fortalezas en la formación académica aunque cabe destacar la falta de formación pedagógica."

D4.C1.P2. [Haciendo relación a la formación que recibió en su Universidad] "La formación es muy completa en cuanto a los contenidos de física, química y biología, esto se evidencia a la hora de trabajar como maestro, pues la gran mayoría de temas que se abordan en el aula son de amplio conocimiento para el docente formado en la licenciatura, la parte pedagógica no me ha causado problemas, aunque vi poco en la universidad, las prácticas permitieron adoptar ciertas técnicas y experiencia que se aplican en el ejercicio profesional." 
Bio - grafía. Escritos sobre la Biología y su Enseñanza. ISSN 2027-1034

Edición Extraordinaria. p.p. 1403 - 1414

Memorias del IX Encuentro Nacional de Experiencias en Enseñanza de la Biología y la

Educación Ambiental. IV Congreso Nacional de Investigación en Enseñanza de la Biología.

Por otra parte, dentro del inventario de preocupaciones y el listado de problemas para docentes principiantes empleados, evidenciamos que las principales dificultades que reconocen los profesores, son presentadas en la Tabla 1.

Tabla 1. Principales preocupaciones de los Docentes Principiantes

\begin{tabular}{|c|c|c|}
\hline Preocupación & Categoría & Docentes Principiantes \\
\hline $\begin{array}{c}\text { Me preocupa que los alumnos } \\
\text { me respeten. }\end{array}$ & $\begin{array}{c}\text { Uno mismo - } \\
\text { Información }\end{array}$ & $\begin{array}{c}\text { Totalmente de Acuerdo: } 21 \\
\text { De acuerdo: } 24\end{array}$ \\
\hline $\begin{array}{c}\text { Me preocupa que mis alumnos } \\
\text { participen e intervengan en las } \\
\text { clases }\end{array}$ & $\begin{array}{c}\text { Impacto - } \\
\text { Consecuencia }\end{array}$ & $\begin{array}{c}\text { Totalmente de Acuerdo: } 30 \\
\text { De acuerdo: } 16\end{array}$ \\
\hline $\begin{array}{c}\text { Creo que puedo hacer } \\
\text { aportaciones importantes a } \\
\text { pesar de mi poca experiencia }\end{array}$ & Tareas - Gestión & $\begin{array}{c}\text { Totalmente de Acuerdo: } 40 \\
\text { De acuerdo: } 10\end{array}$ \\
\hline $\begin{array}{c}\text { Creo que necesito informarme } \\
\text { más para mejorar como profesor }\end{array}$ & $\begin{array}{c}\text { Uno mismo - } \\
\text { Personal }\end{array}$ & $\begin{array}{c}\text { Totalmente de Acuerdo: } 24 \\
\text { De acuerdo: } 18\end{array}$ \\
\hline $\begin{array}{c}\text { Me preocupa que mis } \\
\text { estudiantes aprendan en mis } \\
\text { clases }\end{array}$ & $\begin{array}{c}\text { Impacto - } \\
\text { Consecuencia }\end{array}$ & $\begin{array}{c}\text { Totalmente de Acuerdo: } 32 \\
\text { De acuerdo: } 12\end{array}$ \\
\hline \multicolumn{2}{|c|}{} & \\
\hline
\end{tabular}

Las categorías de la Tabla 1, siguen los planteamientos de la adaptación que hizo Reyes (2011), a partir de las cuatro categorías y siete etapas de Hall et al. (1991). Demostrando que para los docentes principiantes que hasta ahora se han cuestionado, presentan en su mayoría preocupación por su recibimiento en el aula, la imagen que pueden generar en sus estudiantes, los interrogantes que estos harán, entro otros. De igual forma, es evidente que en su mayoría y al tiempo muy significativo para la región, ver que la mayoría consideran que pueden aportar a los procesos pedagógicos de las Instituciones donde laboran, a pesar de la poca experiencia, demostrando su preocupación en la categoría de Tareas y etapa de gestión, que acorde con lo propuesto por Jordell (1987), es importante valorar los niveles de socialización de los docentes, estos pasan por lo personal, el aula, lo institucional y lo social, niveles en los que interactúan y que configuran al profesor, en este caso principiante. 


\title{
Bio - grafía. Escritos sobre la Biología y su Enseñanza. ISSN 2027-1034
}

\section{Edición Extraordinaria. p.p. 1403 - 1414}

\author{
Memorias del IX Encuentro Nacional de Experiencias en Enseñanza de la Biología y la \\ Educación Ambiental. IV Congreso Nacional de Investigación en Enseñanza de la \\ Biología.
}

\section{Docente Principiante: Caso Ana}

El primer acercamiento con la docente principiante, sucedió cuando voluntariamente informó su interés de participar en el proyecto, y bajo consentimiento informado se procedió a aplicar la primera entrevista. En este instrumento se tuvieron en cuenta elementos del conocimiento pedagógico en torno a la enseñanza de las ciencias, el conocimiento de contenido, la actuación de la docente en el aula, el uso del contexto, los mecanismos de evaluación y las estrategias que usa en su práctica, estos elementos aportan a la caracterización del CDC.

La docente, se graduó en el año 2015, ha estado vinculada a 2 Instituciones Educativas, la primera del sector oficial en calidad de plaza provisional para un centro docente del sector rural en el departamento del Huila, y la segunda y actual experiencia, la vive en un colegio del sector privado de Neiva, en donde orienta la asignatura de física con estudiantes de grado $10^{\circ}$ y $11^{\circ}$. Ana, tiene 26 años, integrante de una familia de 11 hermanos, reside en el municipio de Palermo, a 15 minutos de la ciudad de Neiva, casada y con un hijo de 2 años. En su inserción docente, ella define el proceso de la siguiente manera:

C1.E1. [Haciendo referencia al proceso de inserción laboral] "un proceso difícil, en algunos aspectos negativos, ya que el acompañamiento no existió, en el sector público, me fue únicamente entregada la programación y a trabajar, y en el privado, la inducción fue más bien corporativa, empresarial diría yo, no conocí por parte de los coordinadores o del rector, el modelo pedagógico, solo me dieron pautas y directrices de que entregar, cuando y como, pero nunca de cómo hacerlo, sin embargo por otro lado, el proceso lo asumí de manera positiva, recordando mi práctica en la universidad, empecé y creo que con altos y bajos, he hecho las cosas bien, porque se ve en lo que manifiestan mis estudiantes."

En las palabras de la docente, es evidente la necesidad de procesos claros de inducción en esas Instituciones y de acuerdo con resultados de la parte descriptiva, en otras de la región, en donde se haga un acompañamiento a los maestros principiantes, abandonando ideas de inducciones cortas, por discursos de calidad, y por el contrario, más que un "tiempo de prueba", sea un espacio para apoyar al profesorado, se hagan recomendaciones y se permita la inserción oportuna y positiva al nuevo ambiente, en donde las preocupaciones y problemas que se registran en las concepciones de los docentes, se movilicen de totalmente de acuerdo, hacia totalmente en desacuerdo.

En relación a las categorías de CDC, destacamos que Ana, manifiesta un profundo interés por la reflexión que debe hacer todo maestro, autoevaluando su actuar en el aula. Según García (2006), desde el enfoque del profesor investigador, es importante que el profesor sea capaz de cuestionar y reestructurar sus teorías implícitas y las diferentes prácticas que pudo haber aprendido en su formación inicial y que se pueden ir perfeccionando en la 


\title{
Bio - grafía. Escritos sobre la Biología y su Enseñanza. ISSN 2027-1034
}

\section{Edición Extraordinaria. p.p. 1403 - 1414}

\author{
Memorias del IX Encuentro Nacional de Experiencias en Enseñanza de la Biología y la \\ Educación Ambiental. IV Congreso Nacional de Investigación en Enseñanza de la \\ Biología.
}

práctica, pues es ideal ser un profesional autónomo, crítico, investigador y reflexivo sobre su propia práctica profesional.

Estos hallazgos en la iniciación profesional de docentes son positivos, dado que estudios como el de Rivas, Mosquera y Amórtegui (2016), describían la etapa final de la formación docente al interior del programa de ciencias naturales, como un proceso poco encaminado a la reflexión, en donde las aproximaciones a las investigaciones didácticas aunque en ascenso, debían seguirse fortaleciendo. Ahora observamos algunas competencias que le permiten al docente reflexionar sobre la manera como construye un conocimiento, un saber específico en su ejercicio docente, que no sólo lo hace diferente a los demás profesionales, sino a sus colegas de área e institución. De acuerdo con Amórtegui y Correa (2012) y Valbuena (2007), es en la práctica donde se construye el conocimiento didáctico del contenido.

\section{CONCLUSIONES PARCIALES}

Los docentes principiantes reconocen que dentro de las principales dificultades en su proceso de llegada e inserción a las Instituciones Educativas, está la falta de acompañamiento para escoger o seleccionar métodos y demás para el aula, lo que los lleva al ensayo y error, que aunque forma en la práctica, sería menos traumático para el docente principiante, cuando un par docente, aporte a ese proceso.

Por otro lado, dentro de las principales preocupaciones que manifiestan los docentes, encontramos las que hacen alusión a la categoría uno mismo, en la etapa información, al temer por la imagen ante sus estudiantes, las posibles burlas, el error y el rechazo por su comunidad. Así mismo la categoría Impacto, se evidencia como preocupación, cuando en lo que respecta a la etapa consecuencia, el docente principiante, asegura inquietarle el proceso de aprendizaje de sus estudiantes, que sus métodos sean los idóneos y que para el estudiantado no sean aburridores, ya que pueden generar rechazo.

Finalmente es significativo que Ana, como docente principiante considere que la reflexión es un elemento esencial en la labor docente, en su entrevista inicial, manifiesta que el docente debe autoevaluarse, no sólo para ver en que está fallando y mejorar, sino para ponerse en el lugar de sus estudiantes y propender por una relación en donde el aprendizaje es mutuo, una relación horizontal. Este hallazgo, sugiere un progreso en el profesorado del departamento, teniendo en cuenta otros estudios locales, demostrando que los profesores de ciencias naturales, emplean diferentes herramientas de evaluación, valoran su propia práctica y hacen uso del contexto, partiendo de problemáticas ambientales, procesos sociales y culturales, y fenómenos locales que pueden ser abordados desde el salón de clase, para este caso específico, en la enseñanza de la física. 
Bio - grafía. Escritos sobre la Biología y su Enseñanza. ISSN 2027-1034

Edición Extraordinaria. p.p. 1403 - 1414

Memorias del IX Encuentro Nacional de Experiencias en Enseñanza de la Biología y la

Educación Ambiental. IV Congreso Nacional de Investigación en Enseñanza de la Biología.

\section{BIBLIOGRAGÍA}

Amórtegui, E. y Correa, M. (2012). Las Prácticas de Campo Planificadas en el Proyecto Curricular de Licenciatura en Biología de la Universidad Pedagógica Nacional. Caracterización desde la perspectiva del Conocimiento Profesional del Profesor de Biología. Bogotá: Fundación Francisca Radke y Universidad Pedagógica Nacional.

Amórtegui, E. (2011). Concepciones sobre prácticas de campo y su relación con el conocimiento profesional del profesor, de futuros docentes de Biología de la Universidad Pedagógica Nacional (Tesis de Maestría). Bogotá: Universidad Pedagógica Nacional.

Amórtegui, E., Correa, M. y Valbuena, E. (2009). Prácticas de campo planificadas en la formación inicial de futuros profesores de biología y su aporte a la construcción del conocimiento profesional: el caso de primer semestre. Tecné, Episteme y Didaxis, Número extraordinario, 992-1003.

Amórtegui, E., Mosquera, J., Quiroga, A., Dussan, G., Bernal, J. y Dussan, N. (2016). Construcción del conocimiento del profesor de ciencias: un estudio de caso en el marco de la práctica pedagógica de la Universidad Surcolombiana. Revista Tecné, Episteme y Didaxis: TED, Número Extraordinario, 187-194.

Amórtegui, E., Quiroga, A., Dussan, G., Bernal, J., Dussan, N., Rodríguez, H., Huependo, Y. y Mosquera, J. (2015). Construcción del Conocimiento Profesional del Profesor de Ciencias Naturales en el Contexto de la Práctica Pedagógica en la Universidad Surcolombiana, Caso Mario, Resultados Preliminares. Bio - grafía. Escritos sobre la Biología y su Enseñanza, Edición Extraordinaria, 1204-1211.

Bernal, J., Dussan, N., Quiroga A., Dussan, G., Mosquera, J. y Amórtegui, E. (2016). Construcción del conocimiento del profesor de ciencias en el marco de la práctica pedagógica ii de la Universidad Surcolombiana, un estudio de caso Julieta. Revista Tecné, Episteme y Didaxis: TED, Número Extraordinario, 595-602.

Bromme, R. (1988). Conocimientos profesionales de los profesores. Enseñanza de las Ciencias, 6 (1), 19-29.

Castrillón, J., Bahamón, E., Mosquera, J. y Amórtegui, E. (2016). Diseño de unidades didácticas y conocimiento del profesor: un estudio con futuros docentes de ciencias naturales de la Universidad Surcolombiana: resultados preliminares. Revista Tecné, Episteme y Didaxis: TED, Número Extraordinario, 178-186.

Creswell, J. (2005). Educational research: Planning, conducting and evaluating quantitative and quilitative Research (2a. ed.) Upper Saddle River: Pearson Education Inc.

Davis, E., Petish, D., y Smithey, J. (2006). Challenges new science teachers face. Review of Educational Research, 76 (4), 607- 651. 
Bio - grafía. Escritos sobre la Biología y su Enseñanza. ISSN 2027-1034

Edición Extraordinaria. p.p. 1403 - 1414

Memorias del IX Encuentro Nacional de Experiencias en Enseñanza de la Biología y la Educación Ambiental. IV Congreso Nacional de Investigación en Enseñanza de la Biología.

Freire, L. y Fernandez, C. (2015). O professor universitário novato: tensões, dilemas e aprendizados no início da carreira docente. Ciência Educação, 21 (1), 255-272.

García, E (2006). La integración de la teoría con la práctica en la formación inicial del profesorado. Alambique. Didáctica de las Ciencias Experimentales. 47. pp 65- 73.

Grossman, P. (1990). The Making of a Teacher. Theacher Knowledge and Teacher Education. New York: Teachers College, Columbia University.

Hernández, R., Fernández, C., y Baptista, P. (2006). Metodología de la Investigación (Cuarta ed.). México, D.F.: Mc Graw-Hill Editores.

Jiménez, M. M. (2013). Profesor/a principiante de Ciencias Naturales: La configuración de su conocimiento en la inserción profesional (Tesis de Doctorado). Medellín: Universidad de Antioquia.

Jordell, K., (1987). Structural and personal influences in the socialization of beginning teachers. Teaching \& Teacher Education, 3, (3), 165 - 177.

Luft, J., Firestone, J., Wong, S., Ortega, I., Adams, K., \& Bang, E. (2011). Beginning Secondary Science Teacher Induction: A Two-Year Mixed Methods Study. Journal of Research in Science Teaching, 48 (10), 1199-1224.

Marcelo, C., (1991). (Comp.) Aprender a enseñar: un estudio sobre el proceso de socialización de profesores principiantes. Madrid: Centro de Publicaciones del Ministerio de Educación y Ciencia: C.I.D.E.

Marcelo, C., (1999). Estudio sobre estrategias de inserción profesional en Europa. Revista Iberoamericana de Educación, 19, 101 - 143.

Park, S. y Chan, Y. (2012). Mapping Out the Integration of the Components of Pedagogical Content Knowledge (PCK): Examples From High School Biology Classrooms. Journal of Research in Science Teaching, 1-20

Pinto, M. y Gálvez, C. (1996). Análisis documental de contenido. Procesamiento de información. Madrid: Editorial Sintesis.

Ramírez, N. (2016). El proceso de inserción profesional del profesor principiante de ciencias naturales: cuatro casos en Colombia (Tesis de maestría). Universidad de Antioquia, Medellín, Colombia.

Reyes, L. (2011). Profesores Principiantes e Inserción Profesional a la docencia. Preocupaciones, problemas y desafíos. Tesis Doctoral. Sevilla, España: Universidad de Sevilla

Rivas, J., Mosquera, J. y Amórtegui, E. (2016). Estado del arte de los trabajos de grado realizados en el programa de licenciatura en ciencias naturales de la Universidad Surcolombiana (2006-2015): caracterización desde el conocimiento del profesor. Revista Tecné, Episteme y Didaxis: TED, Número Extraordinario, 94-101 
Bio - grafía. Escritos sobre la Biología y su Enseñanza. ISSN 2027-1034

Edición Extraordinaria. p.p. 1403 - 1414

Memorias del IX Encuentro Nacional de Experiencias en Enseñanza de la Biología y la Educación Ambiental. IV Congreso Nacional de Investigación en Enseñanza de la Biología.

Shulman, $L$ (1987). Knowledge and teaching. Foundations of the new reform. Harvard Educational Review, 57 (1).

Shulman, L. (1986). Those who understand: Knowledge growth in teaching. Educational Researcher, 15 (2), 4-14.

Tardif, M. (2004). Los saberes del docente y su desarrollo profesional. Traducción de Pablo Manzano. Madrid: Narcea.

Valbuena, E. (2007). El Conocimiento Didáctico del Contenido Biológico. Estudio de las concepciones disciplinares y didácticas de futuros docentes de la Universidad Pedagógica Nacional (Colombia) (Tesis de Doctorado). Madrid: Universidad Complutense de Madrid.

Veenman, S. (1984). Perceived problems of beginning teachers. Review of Educational Research, 54, (2), 143 -178. 